\title{
Características y expectativas laborales de egresados de la Generación Y de la Universidad Continental, Huancayo
}

\author{
Characteristics and work expectations of graduates of the \\ Generation $\mathrm{Y}$ of the Universidad Continental, Huancayo
}

\author{
Gustavo Loayza Acosta ${ }^{1}$ \\ Escuela Académica Profesional de Economía, Universidad Continental; Huancayo, Perú
}

\section{RESUMEN}

El objetivo fue determinar las características y expectativas laborales de los egresados de la Universidad Continental que pertenecen a la Generación "Y" desde la perspectiva de la Teoría de las Generaciones. La técnica y fuentes de recolección de datos que se utilizó fueron las encuestas y análisis documental. El principal instrumento de recolección de datos fue la Guía de Análisis Documental. La muestra fue de 373 estudiantes de la Universidad Continental que cumplían con las características etáreas de la Generación "Y". Las principales características y expectativas laborales de los egresados de la UC de la Generación "Y" halladas son: El $80 \%$ valora su vida personal y familiar y prefieren trabajar sólo 8 horas diarias; $62 \%$ prefiere trabajar como empleado dependiente mientras construye un negocio personal; $81 \%$ valora los tiempos para viajar, hacer deporte y tener hobbies; $95 \%$ valora tener un horario de trabajo que le permita dedicarse a sus proyectos personales; $77 \%$ prefiere un horario flexible y trabajar sólo las horas necesarias para cumplir con sus responsabilidades; $91 \%$ considera que vale la pena dedicarle su tiempo a un trabajo que lo estimule; $53 \%$ considera que lo que hace más atractivo un centro laboral es si es que le ofrecen una línea de carrera; $44 \%$ afirma que "si hace lo que le gusta o le apasiona" es sinónimo de desarrollo profesional. En conclusión, al decidir permanecer en un centro laboral los dos aspectos más importantes que toman en cuenta son, el $30 \%$ si existe la posibilidad de hacer una línea de carrera y el $29 \%$ por incremento salarial.

Palabras clave: Expectativas laborales, generaciones, generación Y.

\section{ABSTRACT}

The objective was to determine the characteristics and job expectations of the graduates of the Continental University who belong the Generation " $Y$ " from the perspective of the Theory of Generations. The technique and sources of data collection that was used were the surveys and documentary analysis. The main istrument for data collection was the Documentary Analysis Guide. The sample was of 373 students of the Universidad Continental that fulfilled the etareas characteristics of the Generation " $Y$ ". The main characteristics and job expectations of the UC of Generation "Y" found are: the $80 \%$ value their personal and family llife and prefer to work only 8 hours a day; $62 \%$ prefer to work as a dependent employee while building a personal business; $81 \%$ value the times to travel, play sports and have hobbies; $95 \%$ value having a work schedule that allows them to dedícate themselvs to their personal projects; $77 \%$ prefer a flexible schedule and work only the hours necessary to fulfill their responsabilities; $91 \%$ consider it worthwhile to dedícate their time to a job that stimulates them; $53 \%$ consider that what makes a job center more attractive is if they offer a career line; $44 \%$ say that "if you do what you like or are passionate about" is synonymous with professional development. In conclusion, when deciding to stay in a work center, the two most important aspects taken into account are: $30 \%$ if there is the possibility of doing a career path and $29 \%$ by salary increase.

Keywords: Work expectations, generations, generation $\mathrm{Y}$.

Historial del artículo:

Recibido, 12 de abril de 2017; aceptado, 11 de mayo de 2017; disponible en línea, 05 de junio de 2017

* Docente de la Universidad Continental.

Correo: gloayza@continental.edu.pe 


\section{INTRODUCCIÓN}

William Strauss y Neil Howe (1991) fueron los primeros en desarrollar la Teoría de las Generaciones. A pesar de ser un análisis para los Estados Unidos, se ha ido difundiendo su aplicación a nivel mundial. Fueron ellos los que generaron las clasificaciones, definiéndolas como: Generación Silencio, Generación Baby Boomers, Generación X, Generación Y y la Generación Patriota.

El concepto de generación, según la Consultora Gerza (2014) es descrito como aquella característica que es aplicable "a las personas cuya edad es equivalente y vivieron una misma época, en un mismo momento histórico. Por lo anterior presentan características de personalidad similares y comparten creencias, valores y rasgos conductuales". De acuerdo a Long (1997) las generaciones se describen de acuerdo al año de nacimiento de las personas. $Y$ de acuerdo a esta clasificación los que nacieron entre 1926 a 1945 pertenecen a la Generación Silencio, los que nacieron entre 1946 a 1963 pertenecen a la generación Baby Boomer, los que nacieron entre 1964 a 1983 pertenecen a la generación $X$, los que nacieron entre 1985 a 1993 pertenecen a la Generación Y y los que nacieron entre 1994 al 2004 serían la Generación Milenio. De acuerdo a la clasificación realizada por Long, la generación Y tienen al 2017 entre 22 a 36 años.

En el Perú, Arellano (2013), desarrolla un trabajo parecido cuando habla sobre los Estilos de Vida. En el cual el análisis no tiene como base el año de nacimiento sino una forma de "clasificar a la gente, basada en encontrar en la sociedad aquellos grupos de personas que se parecen entre ellas por su manera de ser, de actuar y de pensar, y que son similares en algunas características demográficas o sociales, como podrían ser el sexo, la ocupación, el nivel de modernidad, su actitud hacia la vida $y$, en algunos casos, el nivel de ingreso." Sin embargo, en el Perú las investigaciones sobre la Teoría de las Generaciones se han desarrollado principalmente tomando como universo de estudio poblaciones ubicadas en la provincia de Lima. Siendo escasas y en algunos casos nulos las investigaciones en otras regiones o provincias fuera de Lima.

Existe un estudio realizado por la Universidad Peruana de Ciencias Aplicadas (2014) denominado "La generación Y como fuerza laboral", el cual fue realizado entre los alumnos de dicha universidad y como conclusión principal indican "Existe una clara tendencia al trabajo dependiente versus el independiente, aun cuando existe el presupuesto claro de que los millennials primero tendrían un trabajo dependiente para ganar experiencia y como consecuencia estar suficientemente preparados para realizar un trabajo independiente o tener la propia empresa".

La Teoría de Generaciones cobra relevancia en estos tiempos toda vez que en la mayoría de empresas e instituciones en estos tiempos los trabajadores pertenecen a diferentes generaciones, cada uno con diferentes gustos, preferencias, comportamientos y expectativas. $Y$ esas diferencias hacen que la convivencia entre personas de diferentes generaciones sea cada vez más desafiantes. Sobre lo manifestado líneas arriba, podemos poner como ejemplo las generaciones de los trabajadores del área académica de la Universidad Continental (UC), actualmente en ésta área conviven trabajadores de 4 generaciones diferentes: El Rector (nacido en 1942) pertenece a la Generación Silencio; el decano de Ciencias de la Empresa (nacido en 1959) pertenece a la Generación Baby Boomer; el Director Académico de la Escuela de Contabilidad (nacido en 1970) pertenece a la Generación X; la Asistente Académica de Facultad (nacida en 1986) pertenece a la Generación Y. En este ejemplo, vemos la importancia de conocer las características de comportamiento de cada una de las generaciones para lograr una mejor convivencia laboral y por ende un mejor desempeño laboral de esta área como ejemplo.

Como antecedente a esta investigación determinamos los porcentajes de habitantes por cada una de las Generaciones para la provincia de Huancayo. Para lo cual tomamos en cuenta la estimación al 2015 realizado por el Instituto Nacional de Estadísticas e Informática del Perú (INEI). La información se presenta en la tabla 1 y la figura 1 .

En la tabla 1 y la figura 1 se observa que la Generación "Y" representa el 25,4 \% del total de la población estimada al 2015 de la provincia de Huancayo. La Generación X con el 22,1 \% del total provincial y sólo el 4,3\% pertenece a la Generación Silencio.

Otro dato interesante para el desarrollo de esta investigación es conocer la estructura etárea de los egresados de la UC. En la tabla 2 y figura 2 la población de egresados de la UC que pertenecen a la Generación Y es la mayor con un 77,7 \%, seguida por los egresados de la Generación X con un 21,6 \% y finalmente los de la Generación Baby Bommer con un $0,7 \%$.

Como se aprecia tanto en la provincia de Junín (tabla 1 y figura 1) como en los egresados de la UC (tabla 2 y figura 2) vemos que las población de personas de las Generación $Y$ son la principal población con $25,4 \%$ y 77,7 \% respectivamente. La Generación Y no sólo es la población con mayor porcentaje sino que es la población que encuentra en una transición entre la culminación de sus estudios superiores y búsqueda 
Tabla 1

Distribución de Población por Generación de la provincia de Huancayo.

\begin{tabular}{|c|c|c|c|c|}
\hline \multirow{2}{*}{$\begin{array}{l}\text { Rango } \\
\text { de Edad }\end{array}$} & \multirow{2}{*}{$\begin{array}{c}\text { Cantidad de } \\
\text { Habitantes }\end{array}$} & \multirow{2}{*}{ Porcentaje } & \multicolumn{2}{|c|}{ Clasificación por Generación } \\
\hline & & & Porcentaje & Generación \\
\hline $\begin{array}{l}0-4 \\
5-9\end{array}$ & $\begin{array}{l}47,124 \\
46,530\end{array}$ & $\begin{array}{l}9,4 \% \\
9,2 \%\end{array}$ & $18,6 \%$ & Net \\
\hline $\begin{array}{l}10-14 \\
15-19\end{array}$ & $\begin{array}{l}49,061 \\
51,717\end{array}$ & $\begin{array}{r}9,8 \% \\
10,3 \%\end{array}$ & $20,0 \%$ & Milenio \\
\hline $\begin{array}{l}20-24 \\
25-29 \\
30-34\end{array}$ & $\begin{array}{l}50,443 \\
41,123 \\
36,134\end{array}$ & $\begin{array}{r}10,0 \% \\
8,2 \% \\
7,2 \%\end{array}$ & $25,4 \%$ & Y \\
\hline $\begin{array}{l}35-39 \\
40-44 \\
45-49 \\
50-54\end{array}$ & $\begin{array}{l}31,932 \\
29,713 \\
26,084 \\
23,316\end{array}$ & $\begin{array}{l}6,3 \% \\
5,9 \% \\
5,2 \% \\
4,6 \%\end{array}$ & $22,1 \%$ & $x$ \\
\hline $\begin{array}{l}55-59 \\
60-64 \\
65-69\end{array}$ & $\begin{array}{l}20,118 \\
16,090 \\
12,259\end{array}$ & $\begin{array}{l}4,0 \% \\
3,2 \% \\
2,4 \%\end{array}$ & $9,6 \%$ & Baby Boomer \\
\hline $\begin{array}{c}70 \text { - } 74 \\
75 \text { - } 79 \\
80 \text { y más }\end{array}$ & $\begin{array}{l}8,901 \\
6,539 \\
6,055\end{array}$ & $\begin{array}{l}1,8 \% \\
1,3 \% \\
1,2 \%\end{array}$ & $4,3 \%$ & Silencio \\
\hline Total & 503,139 & $100,0 \%$ & & \\
\hline
\end{tabular}

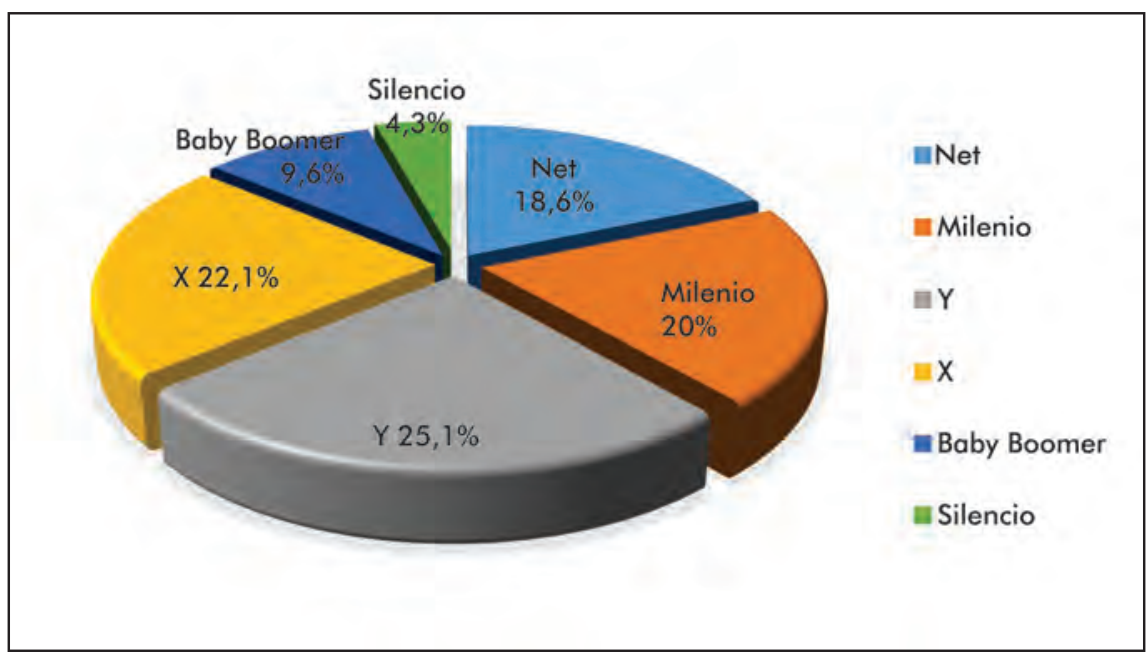

Figura 1. Distribución de Población por Generación de la Provincia de Huancayo.

Tabla 2

Distribución de Egresados por Generación de la Universidad Continental.

\begin{tabular}{cccc}
\hline $\begin{array}{c}\text { Rango } \\
\text { de Edad }\end{array}$ & $\begin{array}{c}\text { Cantidad de } \\
\text { egresados }\end{array}$ & Porcentaje & Generación \\
\hline $22-34$ & 3,788 & $77.7 \%$ & $\mathrm{Y}$ \\
$35-54$ & 1,054 & $21.6 \%$ & $\mathrm{X}$ \\
$54-67$ & 35 & $0.7 \%$ & Baby Boomer \\
\hline Total & 4,877 & $100,0 \%$ & \\
\hline Fuente: OOLA - UC & & &
\end{tabular}

Fuente: OOLA - UC 


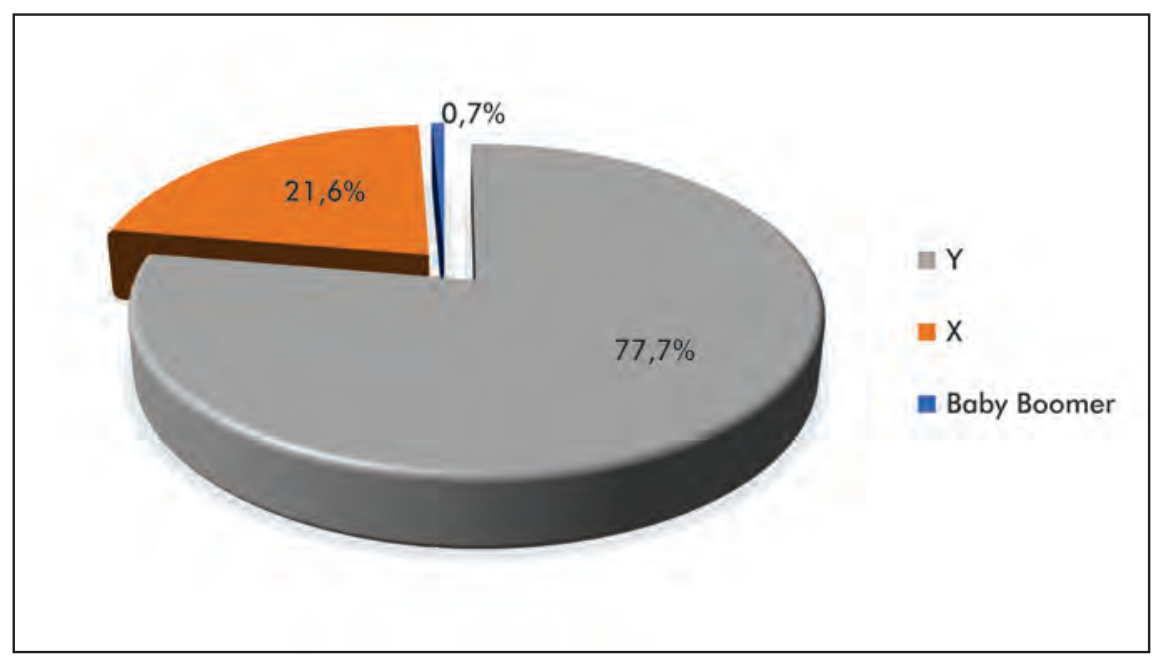

Figura 2. Distribución de Egresados por Generación de la Universidad Continental.

de empleo. En el caso de la UC, se sabe -de acuerdo a la Oficina de Oportunidades Laborales de la UC (OOLA)- que el porcentaje de inserción laboral de los egresados de la UC es del $72 \%$, lo que nos indica que es necesario conocer las características y expectativas laborales de aquellos que pertenecen a la Generación Y para desplegar estrategias que puedan mejorar su empleabilidad.

\section{MATERIAL Y MÉTODOS}

El universo para este estudio son los alumnos egresados de la Universidad Continental que tienen las características citadas por Long (4). De acuerdo a la Oficina de Grados y Títulos de la Universidad Continental el número de egresados a la fecha de esta investigación (Diciembre 2015) es de 4877 egresados, de los cuales aquellos que tienen el rango de edad de la Generación Y son 3788 egresados. Siendo nuestro universo de estudio 3788 egresados por lo que para determinar nuestra muestra estadística usaremos la fórmula para poblaciones finitas. El nivel de confianza para la muestra a calcular será del $95 \%$, la probabilidad de éxito y probabilidad de fracaso será del $50 \%$ porque no tenemos estudios anteriores y el error máximo de nuestra muestra será del $5 \%$.

Los datos para el cálculo de la muestra y la muestra resultante se presentan a continuación:

Aplicando la fórmula de poblaciones finitas:

$$
\frac{N * Z 2 * P * Q}{e^{2 *}(N-1)+Z 2 * P * Q}
$$

Donde:

$\begin{array}{lll}N= & \text { Población } & 3788 \\ Z= & \text { Nivel de confianza } & 1,96 \\ P= & \text { Probabilidad de éxito } & 0,5 \\ Q= & \text { Probabilidad de fracaso } & 0,5 \\ e= & 0,05\end{array}$

El tamaño de muestra para nuestro estudio fue de 349 egresados.

\section{RESULTADOS}

Con el resultado de las encuestas aplicadas a los egresados de la generación "Y" de la Universidad Continental podemos construir un perfil de características y expectativas laborales del egresado de la Universidad Continental que pertenece a la Generación "Y", y estas serían:

a. El $80 \%$ valora su vida personal y familiar y prefieren trabajar sólo 8 horas diarias (figura 3).

b. El $62 \%$ prefiere trabajar como empleado dependiente mientras construye un negocio personal (figura 4).

c. El $81 \%$ valora los tiempos para viajar, hacer deporte y tener hobbies (figura 5).

d. El $95 \%$ valora tener un horario de trabajo que le permita dedicarse a sus proyectos personales (figura 6).

e. El $77 \%$ prefiere un horario flexible y trabajar sólo las horas necesarias para cumplir con sus responsabilidades (figura 7 ).

f. El $91 \%$ considera que vale la pena dedicarle su tiempo a un trabajo que lo estimule (figura 8). 


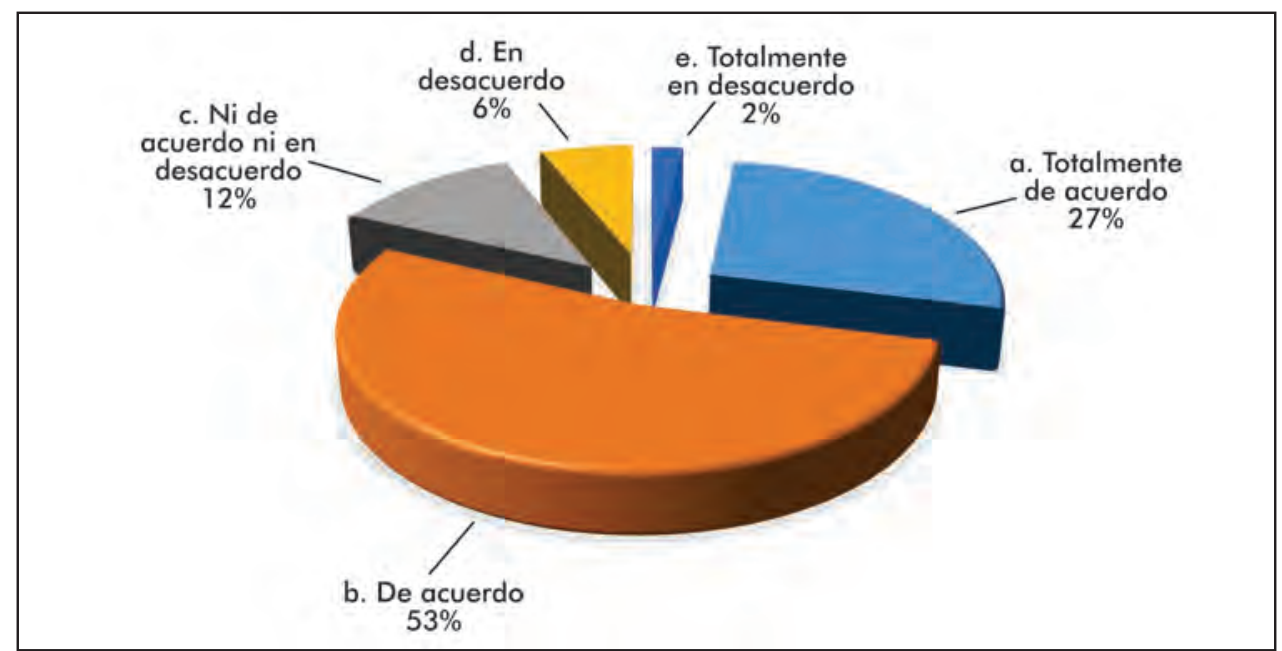

Figura 3. ¿̇Cuán de acuerdo o en desacuerdo esta con la siguiente afirmacion? "Para un joven profesional es importante su vida personal y familiar, y trabajar solo 8 horas"

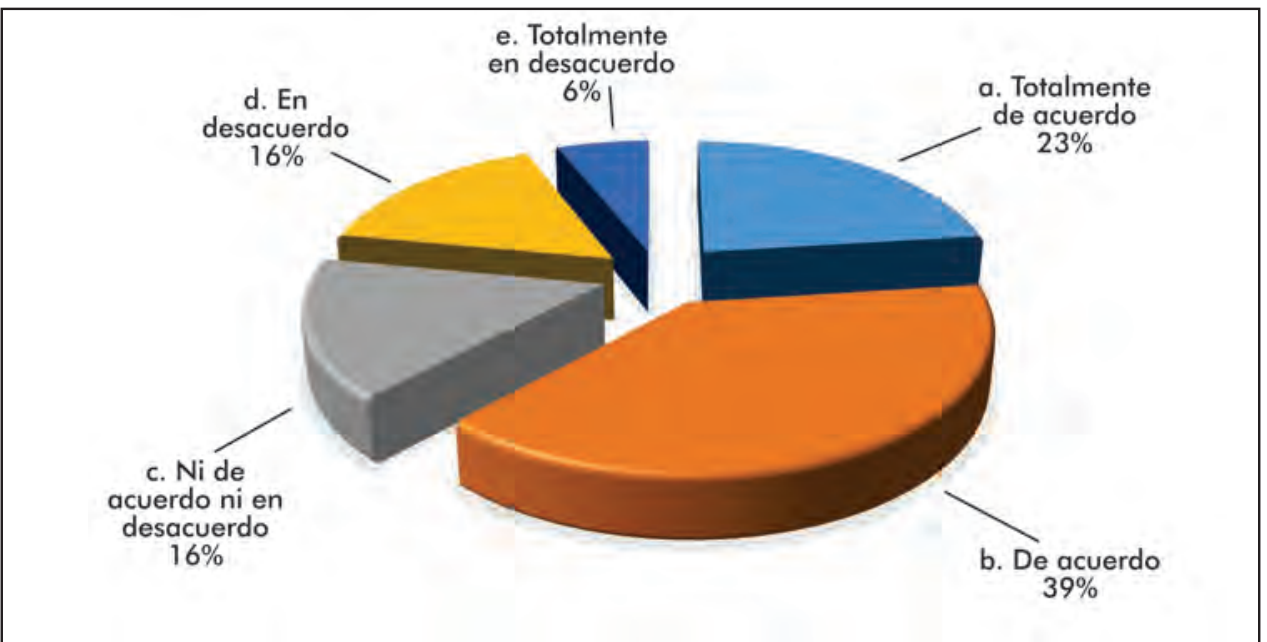

Figura 4. ¿̇Cuán de acuerdo o en desacuerdo esta con la siguiente frase? "Prefiero trabajar como empleado dependiente mientras construyo mi negocio/empresa personal"

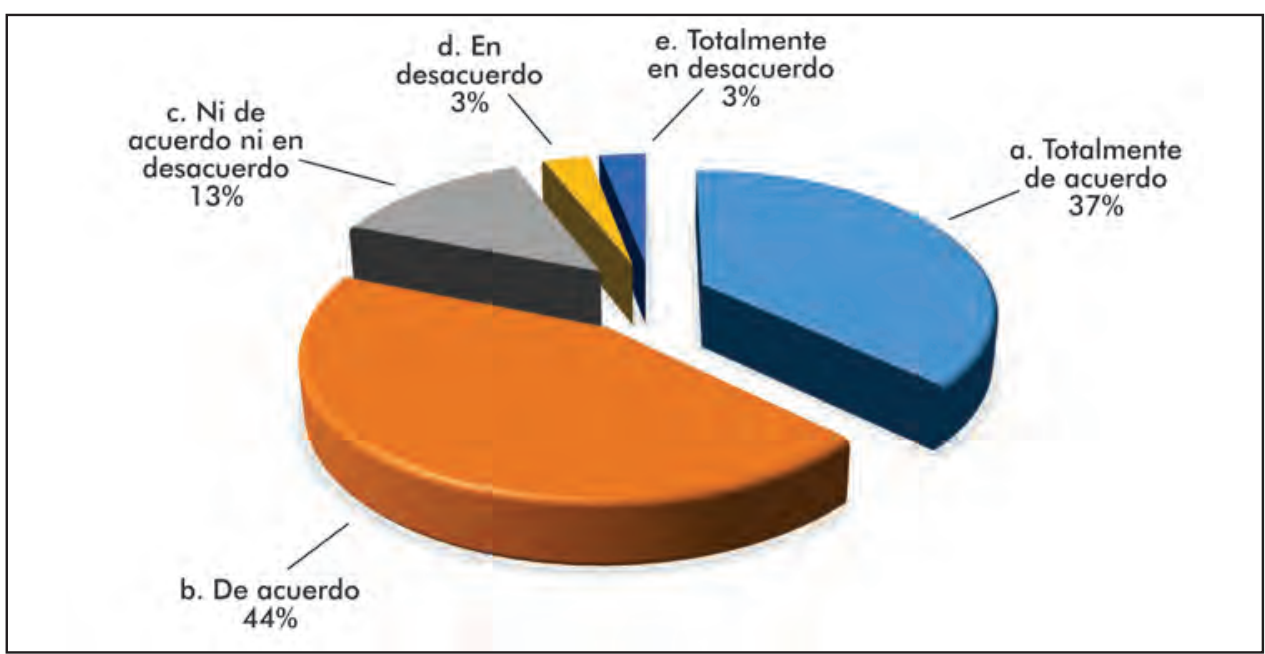

Figura 5. ¿Cuán de acuerdo o en desacuerdo esta con la siguiente frase? "Para un joven profesional Es importante tener tiempo para viajar, hacer deporte, tener hobbies, etc." 


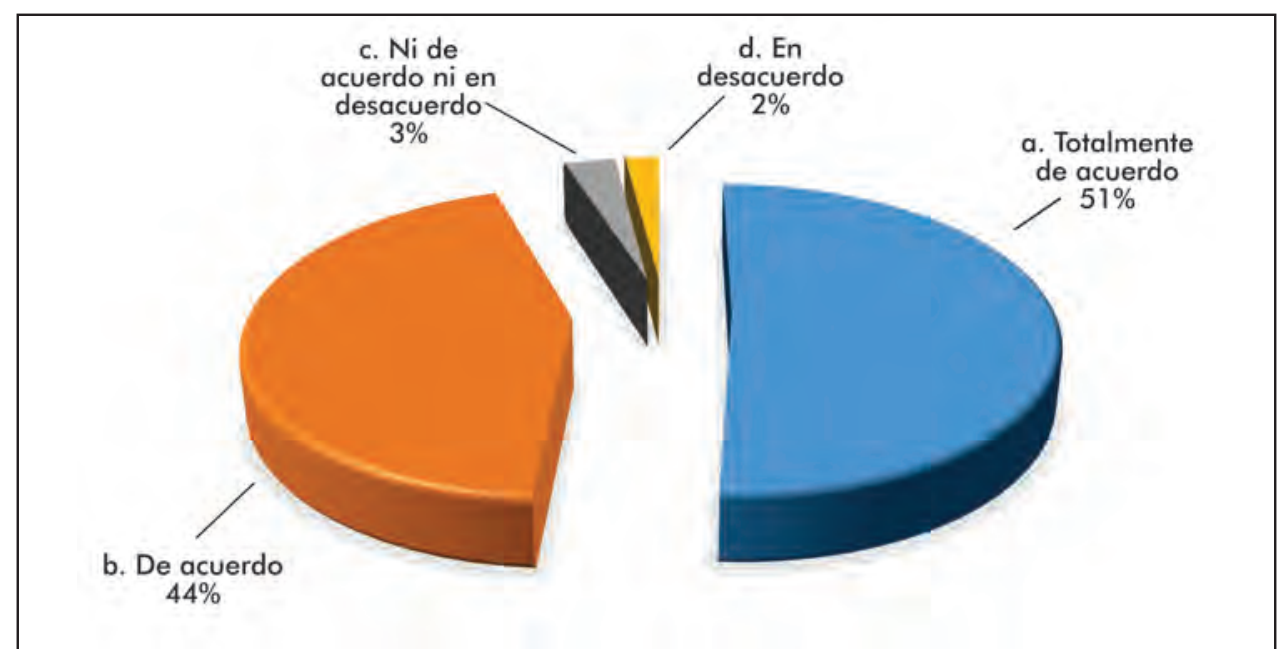

Figura 6. ¿Cuán de acuerdo o desacuerdo esta con la siguiente frase? "Para un joven profesional Vale la pena tener un horario que le permita dedicar tiempo a proyectos personales"

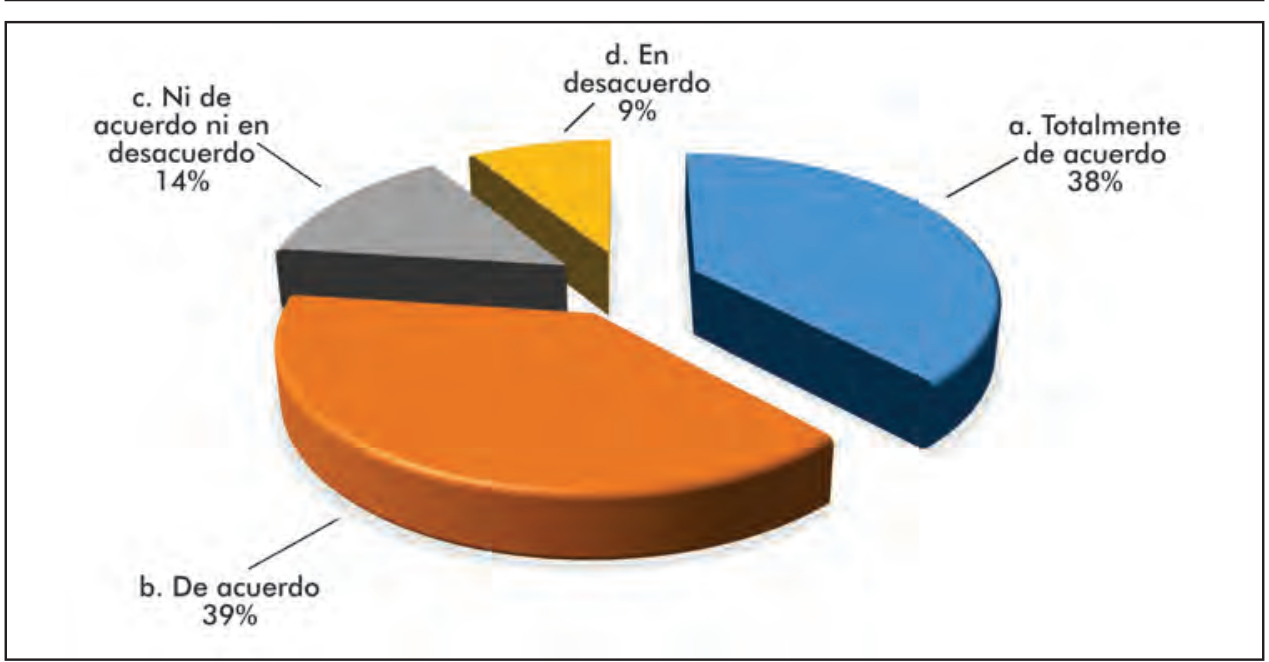

Figura 7.¿̇Cuán de acuerdo o en desacuerdo esta con la siguiente frase? "Para un joven profesional Es importante tener un horario flexible: trabajar solo las horas necesarias para cumplir con sus responsabilidades a tiempo"

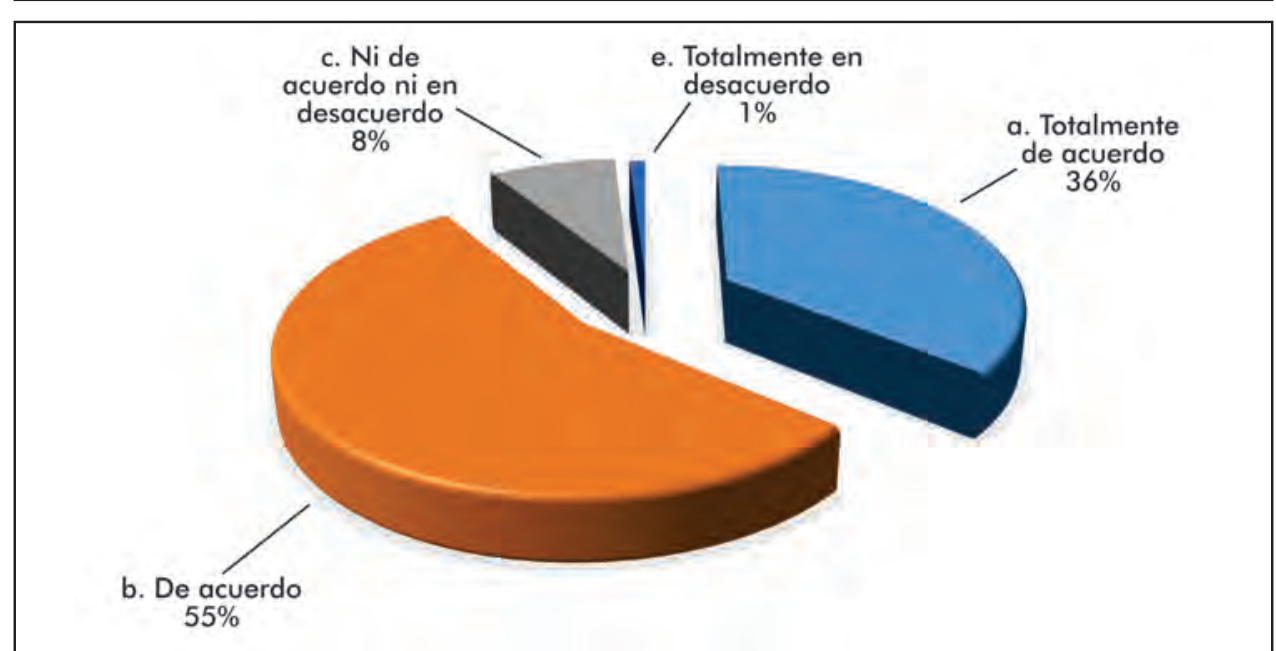

Figura 8. ¿̇Cuán de acuerdo o en desacuerdo estan con la siguiente afirmación? "Para un joven profesional vale la pena dedicarle tiempo personal a un trabajo estimulante" 


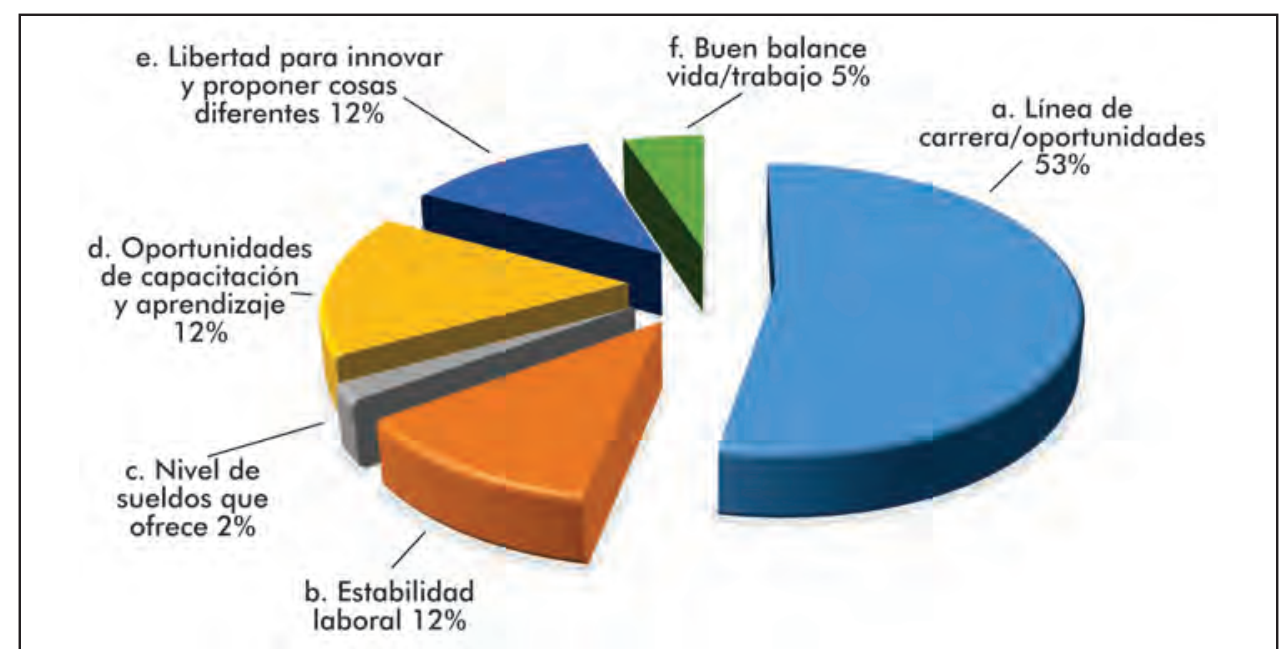

Figura 9. ¿Cuál es lo más importantes para hacer atractiva a una empresa como Centro de trabajo?

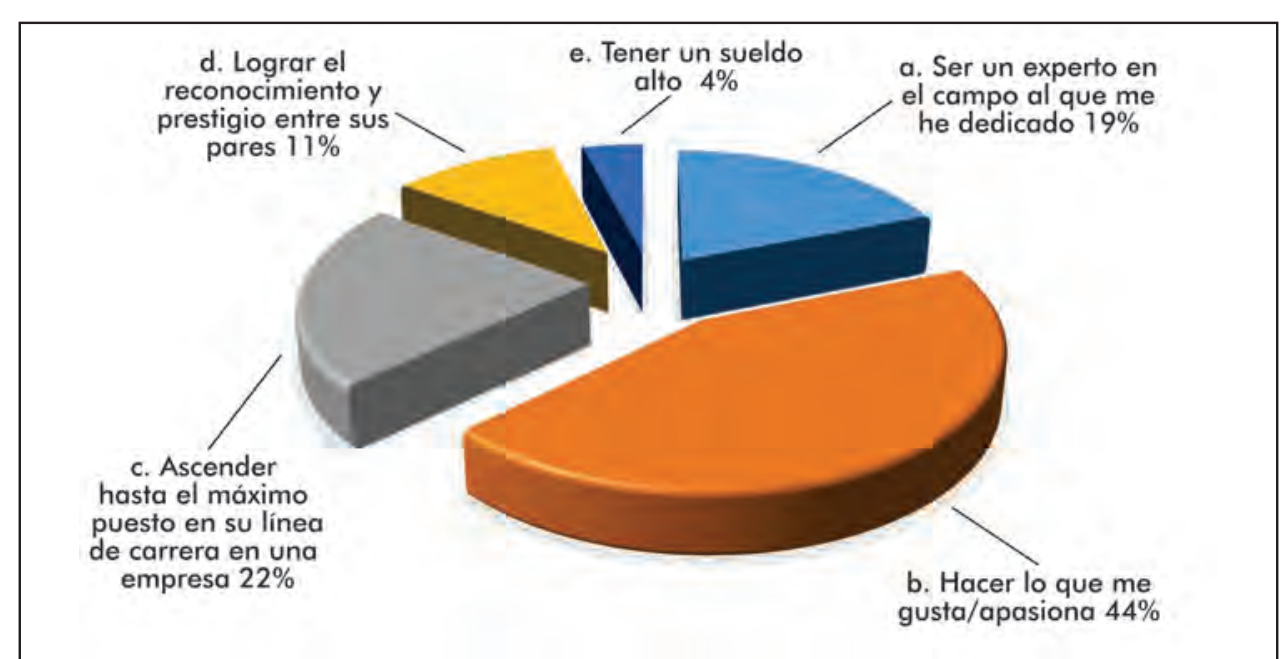

Figura 10. Para usted ¿̇qué significa desarrollarse plenamente en el ámbito profesional?

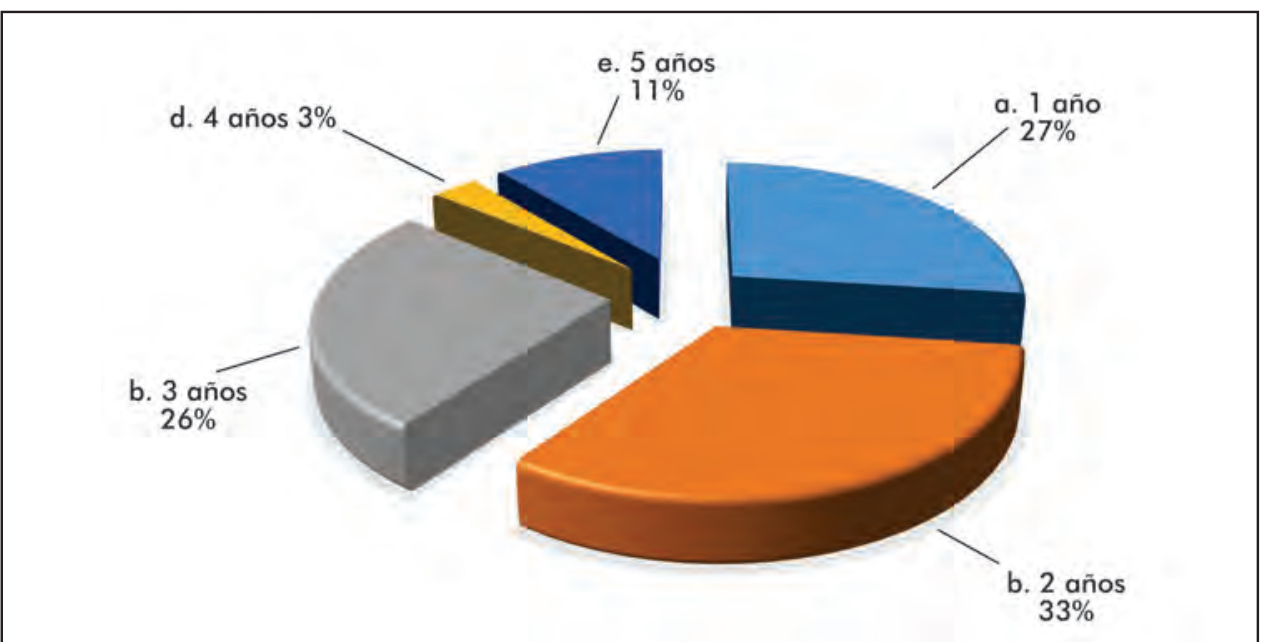

Figura 11. ¿̇uánto tiempo cree usted que debe permanecer una persona en una empresa para poder ganar experiencia necesaria y aprender del negocio? 


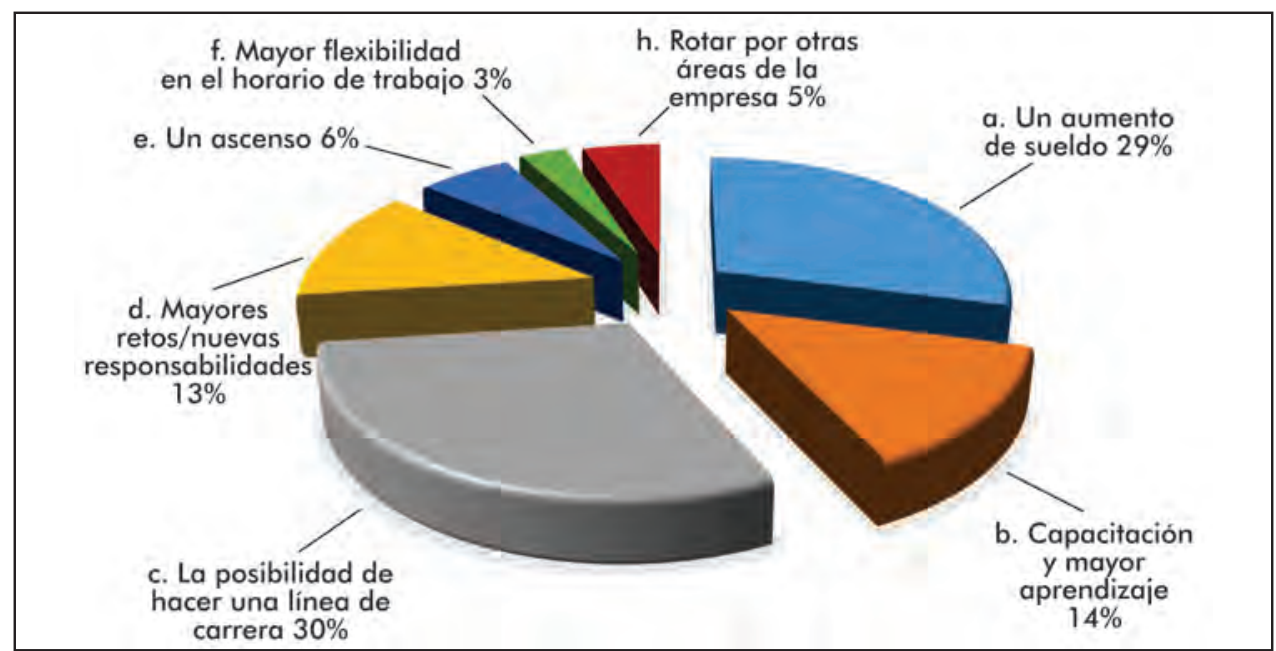

Figura 12. Si tuviera una oferta laboral para cambiar de trabajo, żquÉ aspectos podrían ofrecerle para que decida permanecer en su centro laboral actual?

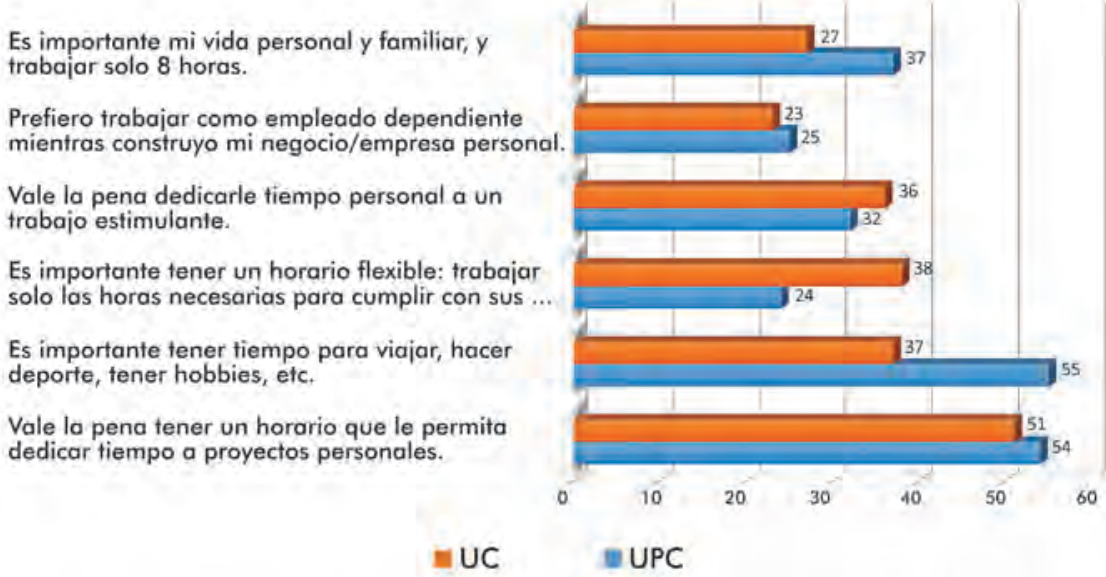

Figura 13. ¿Cuán de acuerdo está con la siguiente frase?: Para un joven profesional... (\%)

Libertad para innovar y proponer cosas diferentes.

Oportunidades de capacitación y aprendizaje.

Estabilidad laboral.

Linea de carrera.

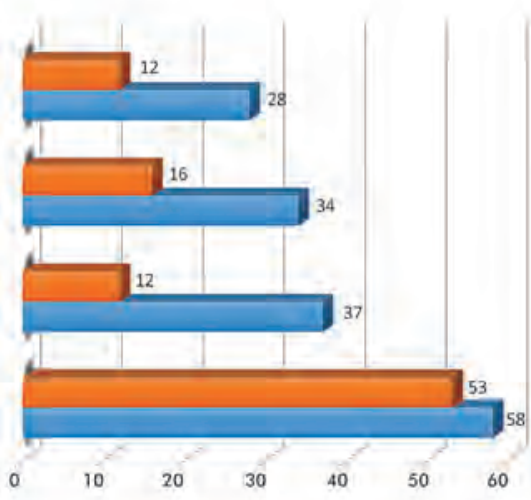

EUC UPC

Figura 14. ¿ Cuáles son las tres cosas más importantes para hacer atractiva una empresa como empleador/centro de trabajo? 


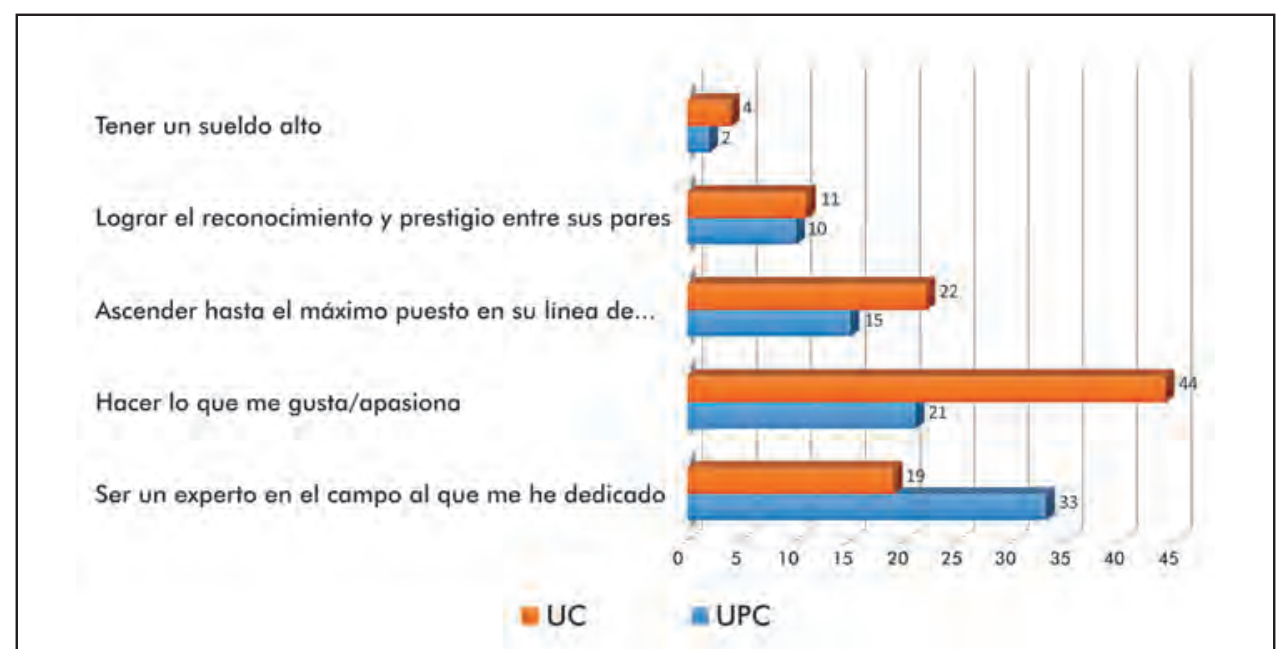

Figura 15. Para usted ¿̇Qué significa desarrollarse plenamente en el ámbito profesional?

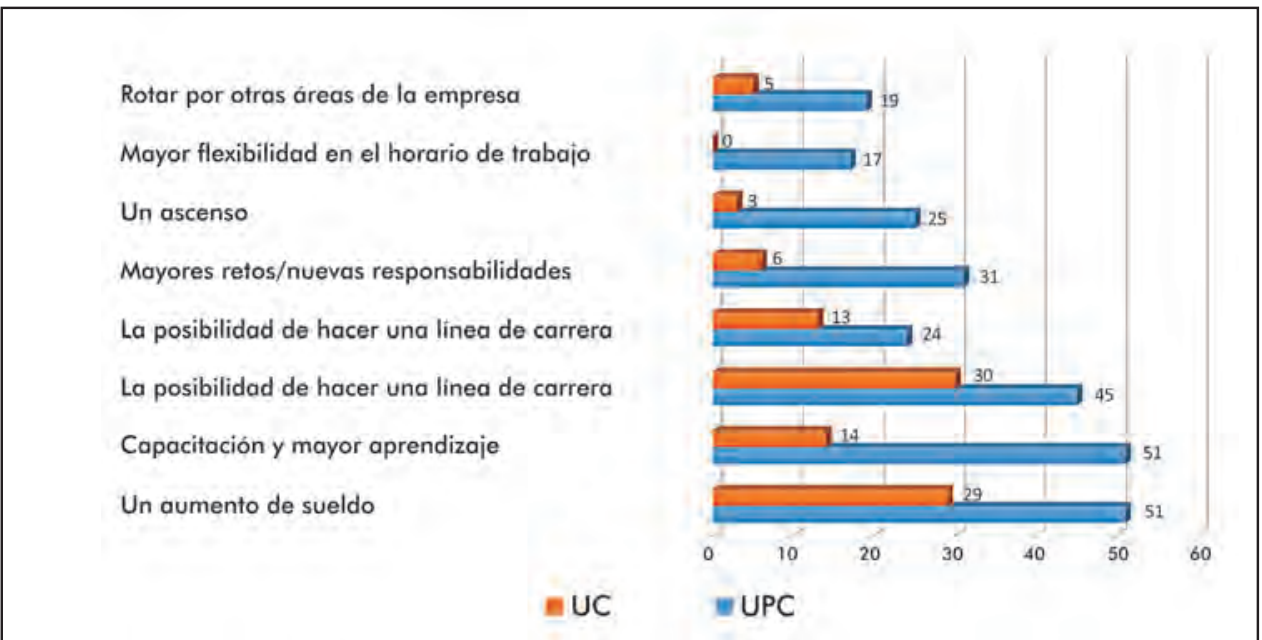

Figura 16. Si tuviera una oferta laboral para cambiar de trabajo, ¿̇ué aspectos podrían ofrecerle para que decida permanecer en su centro laboral actual?

g. El $53 \%$ considera que lo que hace más atractivo un centro laboral es si es que le ofrecen una línea de carrera (figura 9).

h. El $44 \%$ afirma que "si hace lo que le gusta o le apasiona" es sinónimo de Desarrollo Profesional (figura 10).

i. A la hora de decidir permanecer en un centro laboral los dos aspectos más importantes que toman en cuenta son:

- El 30\% si existe la posibilidad de hacer una línea de carrera.

- El 29\% por incremento salarial.

\section{DISCUSIÓN}

Esta investigación tuvo como referencia principal el estudio realizado por la UPC en el año 2014 en el cual se caracterizó los comportamientos de sus estudiantes que pertenecen a la Generación "Y". Ello permite contrastar a ambos tipos de estudiantes pertenecientes a una misma Generación y detallar cuáles son las coincidencias en sus expectativas laborales. En las figuras de la número 13 a la 16 se realiza el análisis de cada uno de los hallazgos realizados.

Ante la pregunta ¿ Qué es lo más importante de un centro laboral? los egresados de la Generación "Y" de la UC y de la UPC coinciden en que lo más importante es un horario de trabajo adecuado a sus necesidades. En contraste lo que menos importancia tiene para ellos es ser trabajador independiente en los primeros años de su vida laboral. 
Frente a la pregunta ¿̇ué es lo que valoran más en un centro laboral? los egresados de la Generación " $Y$ " de la UC y de la UPC coinciden que lo más importante es un centro laboral que les ofrezca una línea de carrera. En contraste, lo que menos importancia tiene para ellos (en diferente grado de intensidad, más en los egresados de la UC) de un centro laboral es la libertad para innovar.

Cuando se les pregunta ¿̇ué significa desarrollo profesional?, para los egresados de la Generación "Y" de la UC implica "hacer lo que lo que le gusta", en cambio para los estudiantes de la Generación Y de la UPC es ser "un experto en su especialidad". En ambos casos el "tener un sueldo alto" pasa a una segunda prioridad.

Ante la pregunta ¿̇ué aspectos podrían ofrecerle para que decida permanecer en su centro laboral actual? los egresados de la Generación "Y" de la UC afirman que mantendrían su centro laboral si le ofrecen línea de carrera. En cambio, para los estudiantes de la Generación "Y" de la UPC es un aumento de sueldo.

Las coincidencias de las características laborales entre los egresados de la Generación "Y" de la UC como de la UPC son:

a. Buscan centros laborales que les permita tener una línea de carrera.

b. Prefieren horarios de trabajo que les permita tener horas líbres para dedicarse a sus proyectos personales.

Las grandes diferencias entre ambos estudiantes que pertenecen a la Generación "Y" son:

a. Cuando se pide la definición sobre "Desarrollo Profesional" los egresados de la UC lo definen como un "trabajo que le apasione". En cambio los estudiantes de la UPC como "experto en su campo laboral o especialidad".

b. Cuando se les pide indicar żpor qué razón cambiarían de trabajo? a parte de un mayor sueldo, los egresados de la UC indican que sería por línea de carrera. Y los estudiantes de la UPC indican que sería por mayor capacitación.

Las conclusiones son:

Las características y expectativas laborales de los egresados de la UC que pertenecen a la Generación "Y" son muy similares a las características de los jóvenes de la Generación "Y" de la UPC. Las características se pueden resumir en las siguientes:

a. Prefiere centros laborales donde pueda trabajar sólo 8 horas, que le permita tener tiempo para espacios de distracción, proyectos personales y diversión.

b. Al inicio de su carrera laboral prefiere trabajar de forma dependiente para generar ahorros y apostar en el mediano plazo por un trabajo independiente.

c. Prefiere trabajar por resultados y no por horarios rígidos.

d. La línea de carrera es algo que será decisivo para elegir un centro laboral así como para cambiar de trabajo, mucho más importante que el nivel salarial.

e. Le gustaría trabajar en un centro laboral realizando labores donde despliegue sus competencias de la especialidad que estudió.

\section{REFERENCIAS BIBLIOGRÁFICAS}

Arellano, R. (2013). Al medio hay sitio. Lima: Editorial Planeta.

Cajal, M (2014) Millennials, Generación X,m Baby Boomers: ¿Cómo se comportan on line? Recuperado 12, junio, 2015 de: https://www. mabelcajal.com/2014/12/millennials-generacionx-baby-boomers-como-se-comportan-online.html/

Coupland, D (1998) Generación X. Editorial Ediciones B. Londres

Gerza Consultora (2014). Comportamiento de las diferentes generaciones. México D.F.

Long, J. (1997). Generación Esperanza, Generación X. Editorial Intervasity

Martinez-Zalce, Graciela (2009). Estados Unidos y Canadá, ¿̇signos conservador hacia el siglo XXI: La generación X: producto del conservadurismo. UNAM, CISAN. Mexico

UPC. (2014) El Monstruo Inexistente ¿̇Qué tan $Y$ es la Generación Y Peruana?. Revista Semana Económica. Lima

Revista de Letras (2012) Generación X la revolución dormida. Recuperado 10, junio, 2015 de http:// revistadeletras.net/generacion-x-la-revoluciondormida/

Strauss W. y Howe N. (1991). Generations: The History of America's Future. New York. 